\title{
Widespread depolarization during expiration: A source of respiratory drive? ${ }^{\text {is }}$
}

\author{
Ravinder Jerath $^{\mathrm{a}, *}$, Molly W. Crawford ${ }^{\mathrm{a}}$, Vernon A. Barnes ${ }^{\mathrm{b}}$, Kyler Harden ${ }^{\mathrm{a}}$ \\ a Augusta Women's Center, Augusta, GA, USA \\ ${ }^{\mathrm{b}}$ Georgia Prevention Institute, Georgia Regents University, Augusta, GA, USA
}

\section{A R T I C L E I N F O}

\section{Article history:}

Received 6 June 2014

Accepted 8 November 2014

Available online $\mathrm{xxxx}$

\begin{abstract}
A B S T R A C T
Respiration influences various pacemakers and rhythms of the body during inspiration and expiration but the underlying mechanisms are relatively unknown. Understanding this phenomenon is important, as breathing disorders, breath holding, and hyperventilation can lead to significant medical conditions. We discuss the physiological modulation of heart rhythm, blood pressure, sympathetic nerve activity, EEG, and other changes observed during inspiration and expiration. We also correlate the intracellular mitochondrial respiratory metabolic processes with real-time breathing and correlate membrane potential changes with inspiration and expiration. We propose that widespread minor hyperpolarization occurs during inspiration and widespread minor depolarization occurs during expiration. This depolarization is likely a source of respiratory drive. Further knowledge of intracellular and extracellular ionic changes associated with respiration will enhance our understanding of respiration and its role as a modulator of cellular membrane potential. This could expand treatment options for a wide range of health conditions, such as breathing disorders, stress-related disorders, and further our understanding of the Hering-Breuer reflex and respiratory sinus arrhythmia.
\end{abstract}

(c) 2014 Elsevier Ltd. All rights reserved.

\section{Introduction}

In addition to gas exchange and oxygenation of the blood, respiration in the lungs can regulate multiple physiological processes throughout the body. Studies of the cardiovascular system, the peripheral nervous system, and the central nervous system provide evidence that respiratory patterns can exert a significant and immediate influence on a wide range of bodily functions, including changes in blood pressure and sympathovagal balance. Patterned respiration can regulate blood pressure and heart rate [1], as well as regulate rhythmic centers of the brain that control cardiovascular output $[2,3]$. While the majority of these findings have demonstrated the impact of respiration on cardiac output and other processes in isolated preparations, little work has focused on respiration's direct influence on membrane potential.

This article reviews the current understanding of respiration as a regulator of cardiovascular physiology and nervous system function. More specifically, we discuss the role of respiratory rhythm and rate on both systemic and local control of these systems. We

\footnotetext{
Funding support: No grants funded the writing of this manuscript.

* Corresponding author at: Augusta Women's Center, 2100 Central Ave, Suite \#7, Augusta, GA 30904, USA. Tel.: +1 706736 5378; fax: +1 7067389922.

E-mail address: rj605r@aol.com (R. Jerath).
}

describe the role of pulmonary stretch receptors (with a focus on slowly adapting receptors) in converting breathing patterns into a functional, multi-faceted, mechanism that allows respiration to communicate with, and direct various aspects of cardiovascular and nervous system physiology. We propose a hypothesis in which inspiration and expiration are associated with transient increases and decreases in membrane potential that may underlie these widespread changes and how these membrane potential changes may underlie the chaotic dynamics of rhythmic breathing.

Many studies focus on the brainstem as the primary modulator of respiration but breathing is affected by many different bodily factors such as airway diameter [4], chest wall mechanics [5], the state of the lungs, chemoreceptors sensitive to hypercapnia and hypoxia [6] and mechanoreceptors in lungs, and intercostal muscles [7]. The brainstem responds to these many peripheral signals and modulates breathing accordingly [8]. We propose that the brainstem responds to peripheral widespread membrane potential changes during respiration, which may be a source of respiratory drive.

\section{Respiratory drive}

Researchers have differing views on the source of respiratory drive. The central pattern generator for respiration is located in 
the brainstem and respiratory neurons in the central pattern generator produce synaptic drive for neurons that control respiratory muscles [9]. It has also been established that respiratory drive derives from detection of high carbon dioxide levels by peripheral chemoreceptors and receptors in the medulla [10]. There is also the theory of hypoxic drive, a form of respiratory drive, in which the medulla detects low levels of oxygen rather than high levels of carbon dioxide, resulting in the drive to breathe [11]. Hypoxic drive was thought to occur in patients with chronically elevated levels of $\mathrm{CO}_{2}$, such as patients with chronic obstructive pulmonary disease (COPD), but recent studies have shown that patients with COPD do not rely on hypoxic drive to breathe [12]. When put on oxygen their $\mathrm{CO}_{2}$ levels may rise due to the Haldane Effect (a reduction in hemoglobin's affinity to bind to $\mathrm{CO}_{2}$ ), and/or ventilation/perfusion imbalance [12].

The urge to breathe is associated with activity in the bilateral insula, frontal operculum, and second somatosensory cortex, which suggests that cortical areas and not just the brainstem are involved in respiratory control [13]. Most respiration studies focus solely on the brain and the role of the brainstem in respiratory drive and control. However, the body's influence on respiration and respiratory drive can seen by the vast afferent nerve feedback [14] and peripheral chemoreceptor feedback that the brain receives from throughout the body [15]. The phrenic nerve is also vital for respiration; if severed, the subject will not be able to breathe without artificial ventilation [16]. Peripheral chemoreceptors detect changes in blood oxygen levels throughout the body and initiate reflexes that maintain homeostasis during hypoxemia [15]. Augmented peripheral chemoreceptors have been shown to be involved with recurrent apnea, congestive heart failure, and some types of hypertension [15].

\section{Hypothesis}

\section{Depolarization as a source of respiratory drive}

We propose that respiration is associated with transient hyperpolarizations and depolarizations of neural and non-neural cells during inspiration and expiration respectively. We hypothesize that membrane potential slightly increases during each inspiration and slightly decreases during each expiration, by way of slowly adapting stretch receptors (SARs) and oxygenation (Fig. 1).

The translation of respiratory patterns into electrical signals in our model occurs primarily via SARs, vagal afferents, and oxygenation and is translated in the brain stem into signals that are conveyed by processes involving the autonomic nervous system (ANS), blood pressure, blood oxygenation, and other hemodynamic processes. Tonic and coordinated polarization (a shift toward hyperpolarization) across groups of neurons has been previously observed. For example, membrane potential signaling in the brain, during non-REM sleep, is dominated by inhibition and hyperpolarization, which likely play an important role during sleep [17]. During nonREM sleep, the cortex, thalamus, hypothalamus, amygdala, and reticular activating system are under widespread inhibitory and hyperpolarizing forces [18]. This widespread inhibition and hyperpolarization occurs during non-REM sleep when breathing is slow and deep [19] breathing is faster and shallower during REM sleep [19]. Although multiple physiological events, such as changes in neurotransmitter signaling, occur during these periods of patterned neural activity, no one physiological mechanism can explain the widespread trend toward partial hyperpolarization.

The widespread hyperpolarization that we propose occurs during inspiration, may lead to the sympathetic nerve inhibition that occurs during slow deep breathing. In addition, the widespread depolarization that occurs during expiration may be similar to the mechanism that is responsible for increased sympathetic nerve activity during apnea. We propose that this widespread partial depolarization, which occurs during exhalation, may be a source for respiratory drive. If a person holds their breath or does not inspire after exhaling, the increasing lack of oxygen leads to further and further minor mitochondrial and cellular depolarizations and increased respiratory drive.

\section{Influence of respiratory rhythm on the autonomic nervous system}

Numerous correlations exist between respiration and the modulation of the sympathetic and parasympathetic nervous systems $[20,21]$. Using several measures of autonomic function, deep breathing techniques (such as pranayama) have been shown to increase parasympathetic activation [22,23]. In contrast, there is a high correlation of increased sympathetic activity with various forms of unstable respiration (i.e. short, rapid breathing and periodic breathing) in patients with heart failure [24]. The respiratory problems in these disorders with widespread autonomic dysfunction demonstrate a strong correlation of respiration with ANS modulation.

Respiration works to regulate properties of muscle contraction via the ANS [25]. In fact, breathing pattern, depth, and possibly starting lung volume all affect sympathetic nerve activity. Reciprocal actions on sympathetic tone occur during deep breathing with inspiration suppressing, and expiration enhancing this input [25]. Suppression of sympathetic input can be enhanced by increases in tidal volume during inspiration, suggesting that SAR mechanoreceptor loading can provide significant input into the regulation of sympathetic control during the resulting period of expiration $[25,26]$. For example, the increase in sympathetic drive in heart failure patients is related to a decreased resting tidal lung volume and a subsequent decrease in the effect of sympathetic inhibition due to lung inflation [25]. Thus, SAR activity represents a major mechanistic link between changes in respiration and corresponding alterations in the regulation of the ANS [27]. Change in sympathetic tone reflects the membrane potential changes brought on by SARs. Inhibitory currents from SARs that reduce retrotrapezoid nucleus (RTN) neuronal firing in the brainstem [28] and sympathetic nerve activity [26] may act as hyperpolarizing currents during inspiration. Respiration's powerful influence on the ANS is apparent in its effects on the cardiac rhythm.

\section{Influence of respiratory rhythm on cardiac rhythm}

Respiration's effects on heart rate and heart rate variability (HRV) are well documented [29]. In addition, SAR activation through vagal afferents may regulate cardiovascular function and cardiac output through more direct mechanisms than discussed above. Although a direct cause and effect relationship has not been well established, variability in heart rate frequency has been employed as a method to differentiate sympathetic (low frequency) from parasympathetic (high frequency) autonomic function [30]. Respiratory sinus arrhythmia (RSA), a naturally occurring variation in heart rate that occurs during breathing cycles, is characterized by shortening of the beat-to-beat interval during inspiration and lengthening during expiration [31]. This manner of precise timing between heart beat and respiration could improve the efficiency of pulmonary gas exchange [31].

Ventricular repolarization also undergoes modulation during breathing [32]. In one study, normal ventricle electrical activity was measured in patients undergoing a heart procedure (i.e. radiofrequency ablation for supraventricular arrhythmias). In these patients, ventricular repolarization time decreased during inspiration and increased during expiration [32]. In this study, the right ventricular endocardium became more hyperpolarized with each 


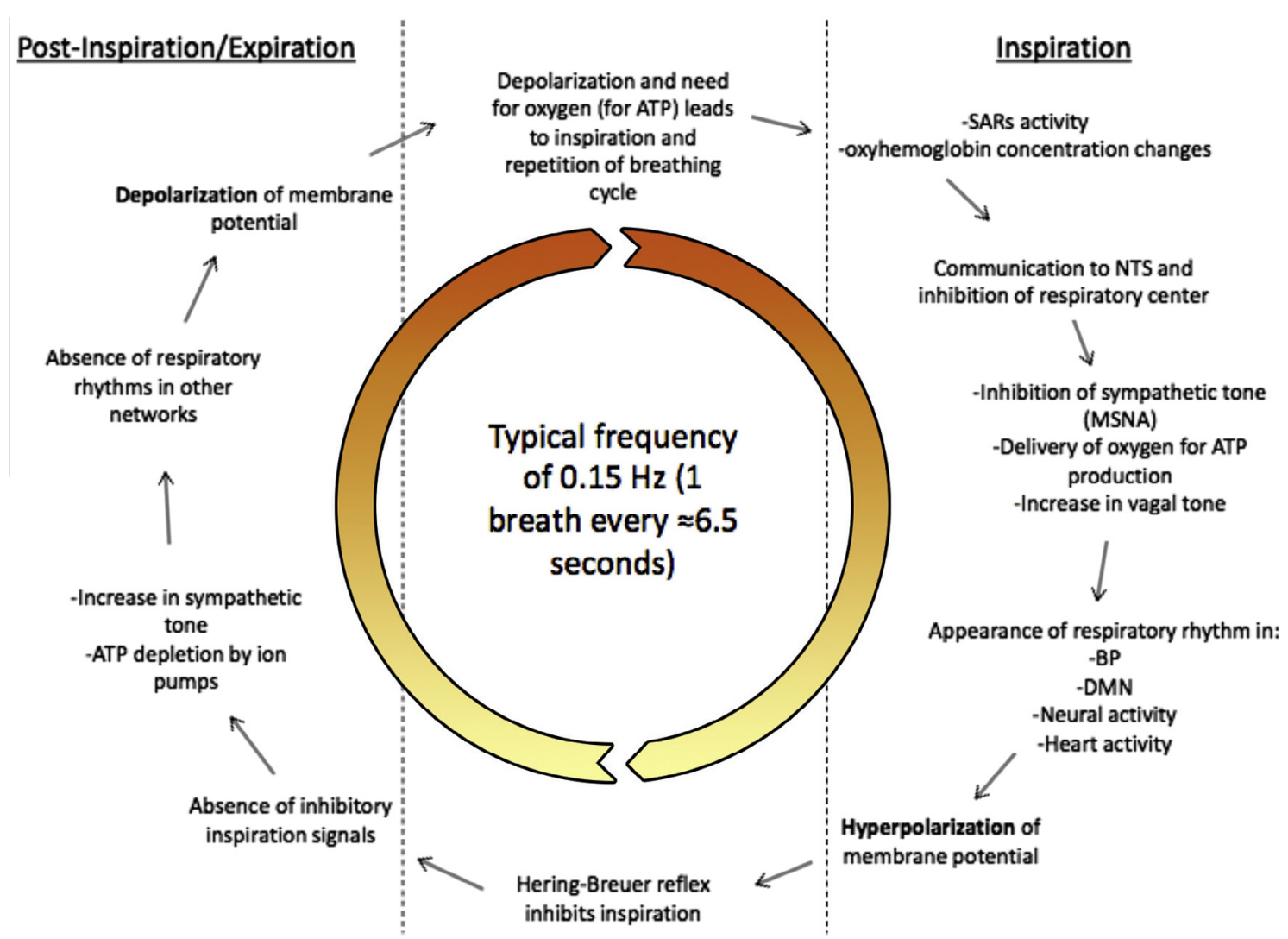

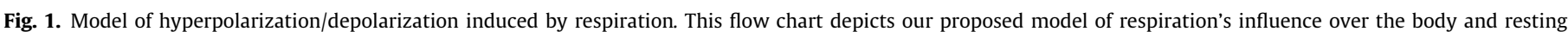

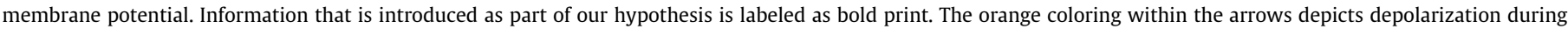

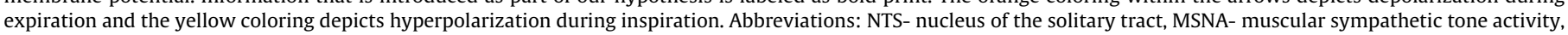
DMN- default mode network. (For interpretation of the references to colour in this figure legend, the reader is referred to the web version of this article.)

beat during inspiration and more depolarized with each beat during expiration, suggesting that ventricular action potential duration varies with the breathing cycle, independently of heart rate variation. In addition to cardiac rhythm, respiration also has profound effects on blood pressure and vascular resistance.

Influence of respiratory rhythm on blood pressure and peripheral resistance

The substantial influence of respiration on cardiac activity via the ANS could also allow respiration's influence to extend to processes governed by the heart. For instance, the influence of respiration on blood pressure (BP) oscillations could be viewed as a mechanism of conveying respiratory activity in the body. Arterial BP levels throughout the body oscillate at a frequency that is dependent upon other physiological systems including heartbeat, respiration, and intrinsic myogenic vascular muscle tone. BP oscillations of $0.1 \mathrm{~Hz}$, commonly referred to as Mayer waves, are associated with sympathetic nervous activity $[2,33]$. In contrast, BP oscillations at a frequency of $0.25-0.3 \mathrm{~Hz}$ correlate with respiratory rhythms [33]. The synchronization of BP oscillations with respiration supports the theory of a baroreflex mechanism as an explanation for RSA. However, baroreflex R-R interval responses can be observed prior to changes in BP [32,34], which suggests the involvement of a possible mechanism that is driven by afferent SARs through the nucleus tractus solitarii (NTS). BP increases during inspiration, reaching its peak just prior to expiration, and falls during the expiration process [32]. One important implication of BP-respiratory synchronization may be the utilization of BP oscillations to relate breathing patterns throughout parts of the body. The effects of respiration on vasculature and circulation, lead to changes in cerebral blood flow and levels of oxyhemoglobin.

Influence of respiratory rhythm on the oscillations of oxyhemoglobin in the prefrontal cortex and cerebral vessels

Growing evidence suggests that oxygen delivery may be one mechanism in which BP could act to relay information on patterned respiration throughout the body. Oxyhemoglobin concentration in the blood is determined by gas exchange in the lungs (breathing) and recent studies suggest that arterial BP facilitates the patterned delivery of oxyhemoglobin to various areas in the body, including the brain. For instance, spontaneous changes in cerebral blood flow have been strongly linked with changes in arterial BP [35]. Fluctuations in cerebral oxyhemoglobin concentration are influenced by heart rate and oscillations in BP and overlap, to a large extent, with frequencies in respiratory rhythm [36]. Oscillations in concentrations of oxyhemoglobin in the prefrontal cortex, with a frequency of approximately $0.07-0.13 \mathrm{~Hz}$, correlate with respiration in response to specific breathing patterns [37]. Finally, oscillations in the flow of cerebrospinal fluid (frequencies between 0.15 and $0.6 \mathrm{~Hz}$ ) overlap with those in cerebral blood flow [38]. Respiratory rhythms also directly influence neuronal membrane potentials in the olfactory bulb and the RTN.

Influence of respiratory rhythm on olfactory neuronal firing pattern of olfactory tuft cells and retrotrapezoid nucleus

The function of the olfactory bulb provides an interesting example of the relationship between the physiology of neural activity and respiration. Oscillations of membrane potential and neuronal 
firing patterns of mitral and tuft cells in the olfactory bulb are regulated by the frequency of respiration [39]. Studies show that the membrane potential of these cells oscillate in synchrony with respiration, in the presence and absence of an odor stimulus [40,41]. Furthermore, when hyperpolarization is induced, mitral and tuft cells exhibit a greater synchronized membrane potential oscillation response to respiratory patterns [40].

Neurons of the RTN, which is located in the rostral medulla oblongata, innervate respiratory pattern generator areas of the brainstem [42]. The RTN, a central chemoreceptor site responsible for the 'drive' to breathe [43], is important in maintaining involuntary breathing during anesthesia [44] and may be essential for involuntary breathing during sleep [45]. Chemoreceptors in the RTN receive inhibitory input from stretch receptors in the lungs, most likely SARs, during lung inflation [28]. Inhibition of the RTN lessens the respiratory chemoreflex whereas stimulation increases respiratory rate [42]. Respiration, particularly inspiration, modulates RTN neuronal firing via SARs [28] and in turn may affect respiratory pattern generation in the brainstem [46]. In other words, RTN neurons are hyperpolarized by SARs during inspiration. Inspiration modulates the ANS and inhibits sympathetic drive likely via SARs.

\section{Slowly adapting stretch receptor rhythms modulate the respiratory center in the brain stem}

Pulmonary stretch receptors, which include both rapidly and slowly adapting types, are found throughout the respiratory tract and are responsive to mechanical, noxious (chemical) and inflammatory stimuli [27]. SARs reside predominantly in the tracheobronchial tree and peripheral lung where they respond to changes in smooth muscle contraction during inspiration. Although both rapidly adapting stretch receptors (RARs) and SARs project to medullary structures in the brain stem, their effects on physiological processes in the body may vary to a large extent. Sympathetic nerve activity is inhibited during inspiration, most likely due to modulation by pulmonary stretch receptors [26]. This sympathoinhibitory effect is enhanced during slow, deep breathing [26]. SARs hyperpolarize and produce inhibitory impulses posthyperinflation [47] and SARs influence breathing pattern, heart rate, vascular resistance, and smooth muscle tone [48]. The mechanism by which SARs control these functions is not well understood [48]. These inhibitory impulses may be the mechanism by which SARs influence the autonomic nervous and cardiovascular systems. We propose that inhibition by SARs, when associated with oxygenation, leads to hyperpolarization of neural and nonneural tissues. Although SAR projections have diverse targets in the brain stem, they are considered the main pulmonary mechanoreceptor responsible for preventing excessive lung inflation in response to smooth muscle activity that occurs during inspiration [27]. This well-characterized reflex between the lung and brain stem is called the Hering-Breuer inflation reflex [27]. Once activated, SARs and RARs induce action potentials through projections that run along the vagus nerve and synapse on second order neurons in the caudal portion of the nucleus of the solitary tract (NTS) in the medullary region of the brainstem $[49,50]$. Current evidence points to SAR activation as a major link between the mechanical stimulus induced by breathing and the physiological responses induced by this stimulus during respiration.

SAR projections form monosynaptic connections with two subpopulations of neurons, the pump cells (P-cells) and inspiratory- $\beta$ $(\mathrm{I} \beta)$ cells [51]. Studies have demonstrated that P- and I $\beta$ cells display a phasic firing pattern that mimics SAR activity and tracks pulmonary oscillations [52,53]. This suggests that these neuronal populations act in some capacity to relay patterns of respiration from SARs in the lung to regions of the central nervous system
(CNS) in which third order SAR neurons reside. Therefore, the NTS may act as a staging area in the brain stem that receives respiratory input and translates respiratory patterns (through SARs and perhaps other tracheobranchial fibers) into a signal that is understood by multiple neuronal populations in areas of the CNS. To better understand the influence of respiration and SARs on the ANS and membrane potentials we must examine respiration at the cellular level.

\section{Influence of respiration on the cellular level}

$\mathrm{Ca}^{2+}$ signaling coordinates mitochondrial ATP production with cellular work so that energy conversion and utilization are equal [54]. Rate of respiration is directly proportional to the level of work $[55,56]$. During exercise both rate of respiration (breathing) and glucose metabolism increase in proportion to the exertion level during exercise to meet increased demands [57]. There is only enough ATP stored for $1-2 \mathrm{~s}$ of work so ATP must be rapidly synthesized according to demand [57]. In the absence of oxygen, anaerobic ATP supply pathways are initiated but these can only sustain demands of ATP for the brain cells and tissue for a few minutes, or a few hours for skeletal muscle [58]. It has been well established that oxygen is vital for aerobic respiration yet the direct relationship of breathing with ATP production has not been well recognized. These processes and changes that occur at a cellular level have not been linked to inspiration and expiration. During inhalation, oxygenation of tissues occurs within the lungs and then oxygen is carried by red blood cells throughout the body, oxygenating tissues. This oxygenation leads to the first stage of cellular respiration, glycolysis. Protons from the high-energy bonds of $\mathrm{NADH}$ and $\mathrm{FADH}_{2}$ that are pumped across the mitochondrial membrane [59] lead to membrane hyperpolarization [60], in which the interior matrix voltage of the mitochondria is more negative [61] At the same time, during inhalation, the inflation of alveoli and the trancheo-bronchial tree may cause SARs to send hyperpolarization impulses to the brain [47]. Protons translocate across the membrane to facilitate establishing a transmembrane potential [62].

Exhalation's main purpose is to rid the body of carbon dioxide, a by-product of cellular respiration. The changing levels of oxygen and $\mathrm{CO}_{2}$ in inhaled and exhaled air suggest that cellular respiration may slow during exhalation due to the decreased presence of oxygen. One study found spontaneous mitochondrial membrane potential changes in cultured neurons [63]. The researchers proposed that these partial, transient depolarizations may reflect the mitochondria alternating between active and inactive oxidative phosphorylation [63]. Other studies have also shown mitochondrial membrane potential changes in isolated mitochondria [64,65], and in mitochondria within different cell types, such as cardiomyocytes [66], vascular endothelium [65], and pancreatic B-cells [67]. Buckman and Reynolds found that oligomycin, which inhibits ATP-synthase, decreased the number of fluctuations, suggesting that the fluctuations are related to oxidative phosphorylation [63]. The researchers also performed several experiments to eliminate other explanations for these depolarizations and repolarizations. This study supports our hypothesis that inspiration/oxygenation, leads to hyperpolarization of the mitochondrial membrane during cellular respiration and that the membrane is hyperpolarized during active oxidative phosphorylation and depolarized during expiration, when oxidative phosphorylation is inactive or reduced. We emphasize that hyperpolarization, which occurs as a result of inspiration, is only partial hyperpolarization. Maximal mitochondrial hyperpolarization has been associated with the "shutting off" of mitochondria and subsequent depletion of ATP that can be seen in cancer [68]. 
Mitochondrial potential is generated by a proton gradient, and inhibition of the electron transport chain would limit proton pumping and lead to depolarization [69]. In the presence of oxygen, $\mathrm{F}_{1} \mathrm{~F}_{0}$-ATPase is the site of ATP production but during anoxia it begins to run backwards, pumping protons from the matrix to maintain the membrane potential [70]. This depletes ATP rather than producing it [70]. In fact, $\mathrm{F}_{1} \mathrm{~F}_{0}$-ATPase requires a membrane potential to induce a rotary torque because it is driven by proton reentry [71]. In addition, hypoxia has been shown to inhibit the electron transport chain [72]. These studies suggest that lack of oxygen may lead to depolarization and reduced levels of oxygen may lead to partial depolarization. Studies have shown that hypoxia leads to depolarization in different types of rat cells $[73,74]$, as well as depolarization of mitochondria [75,76]. This hypoxic depolarization of mitochondria appears to be caused by decreased electron transport chain activity due to lack of oxygen [76]. In addition, this mitochondrial depolarization has been shown to precede hypoxic spreading depression, suggesting that the mitochondrial membrane potential can influence the cell's membrane potential [76], consistent with our hypothesis. During expiration the lowering levels of oxygen may cause inhibition of oxidative phosphorylation and subsequent depolarization followed by polarization (hyperpolarization) during inspiration and resupply of oxygen. We do not suggest that the degree of membrane depolarization, that may occur during expiration, is anywhere near the level that occurs during hypoxia. We propose that decreases in oxygen, at the levels measured during expiration, may lead to slight increases in the degree of membrane depolarizations. Previous studies discussed, found that transient mitochondrial membrane hyperpolarizations and depolarizations may indicate active and inactive states of oxidative phosphorylation [63], consistent with our proposed hypothesis, though they did not directly link these processes with oxygen intake. We propose that these mitochondrial membrane potential changes extend to the membrane of the cell, leading to slight hyperpolarizations and depolarizations of the cell membrane. The importance of these membrane potential changes and the influence of respiration on the body and brain can be better understood by examining the physiological and neurological changes that occur in breath-holding and breathing disorders.

\section{Breath-holding and breathing disorders}

Breath holding does not stop the central respiratory rhythm; the rhythm continues and is merely suppressed by voluntarily holding the chest at a chosen volume [77]. In addition, the length of time a person is able to hold their breath is increased by bilateral paralysis of the phrenic or vagus nerve, suggesting that stimulation of peripheral diaphragm muscle chemoreceptors may contribute to the breakpoint of breath holding more than previously thought [77]. Duration of breath-holding has been shown to be reduced by factors that increase feedback from diaphragm afferents, such as tonic diaphragm activity, or factors that increase the central respiratory rhythm, such as hypoxia or hypercapnia, decreasing lung volume, or increased metabolic rate [77]. During breath-holding, respiratory drive is stimulated when the breaking point is reached, likely due to elevated levels of $\mathrm{CO}_{2}$, there is a growing urge to breath and involuntary contractions of respiratory muscles known as involuntary breathing movements (IBMs) [78]. These IBMs have been shown to increase cerebral oxygenation during this struggle phase of apnea [78].

Involuntary apneas, occurring during sleep, as in obstructive sleep apnea (OSA), have been shown to cause long-term health problems such as hypertension, stroke, coronary artery disease, or cardiac arrhythmias [79]. Defects in respiratory control are known to be involved in the pathogenesis of OSA [80]. Daytime hyperten- sion caused by OSA is due to increased chemoreceptor sensitivity causing excessive sympathetic vasoconstriction, increased superoxide production, and inflammation of vessels [79]. OSA can also lead to hypoxia-induced neural damage resulting in impaired memory and concentration [79]. Intermittent hypoxia can affect insulin sensitivity and glucose regulation but it is unknown whether OSA attributes significantly to metabolic syndrome or whether these are more attributable to obesity [79]. Experiments on voluntary apneas have shown acute and lasting effects on autonomic regulation of the cardiovascular system and increased sympathetic nerve activity caused by hypoxia $[81,82]$. Hypoxia has also been shown to induce hypoxic spreading depression-like depolarization in areas throughout the brain, including hippocampal [83] and cortical tissues [84]. We propose that this well-documented hypoxic depolarization occurs on a much smaller scale during each expiration. If a person does not inspire after exhaling, and holds the breath, this depolarization becomes more and more pronounced, increasing the drive to breathe via widespread depolarization.

In addition to hypoxia, low levels of $\mathrm{CO}_{2}$, like those seen in hyperventilation can also cause negative effects [85]. Hyperventilation leads to an increase in organic acids and causes changes in metabolism [86]. The formation of these organic acid-calcium complexes decreases the calcium activity at the cell membrane [86]. This suggests that cells become unstable and depolarize during hyperventilation because there is less blood calcium available at the membrane. Hypocalcemia and/or alkalosis, caused by hyperventilation can lead to tetany, a disorder in which increased neuronal excitability causes painful muscle cramping and spasming [85]. Hyperventilation can lead to hypoxia [87], increased heart rate, peripheral and cerebral vasoconstriction with reductions of blood flow to the brain by up to $30 \%$, and interference with tissue oxidation [88]. These studies demonstrate the delicate balance that must be maintained between oxygen, $\mathrm{CO}_{2}$, and membrane potentials. Detection of elevated $\mathrm{CO}_{2}$ levels is likely a factor in respiratory drive but we propose an additional mechanism in which widespread partial depolarization may also be a source of respiratory drive.

\section{Evaluation and consequences of the hypothesis}

The current understanding of respiratory drive is that respiratory centers in the brainstem control rate and depth of respiration via movements of the diaphragm and other respiratory muscles. Peripheral chemoreceptors detect elevated levels of $\mathrm{CO}_{2}$ in the blood which stimulate the respiratory center to stimulate respiratory muscles to breathe [10]. There is also the debated existence of hypoxic drive, in individuals with compromised respiratory function, in which the body uses oxygen chemoreceptors rather than $\mathrm{CO}_{2}$ chemoreceptors [11]. We propose a form of respiratory drive that involves changing levels of oxygen but does not involve oxygen chemoreceptors as specified in the hypoxic drive theory. The proposed mechanism involves widespread partial depolarization of cells throughout the brain and body during exhalation due to decreased oxygen and increased sympathetic drive. Although we do not challenge the major contributions of $\mathrm{CO}_{2}$ detection to respiratory drive, we propose that the widespread depolarization of cells is also an important contributing factor. This mechanism could be examined by using microsensors to measure oxygen tension and action potential simultaneously in various cell types and may be more pronounced in cells with higher metabolic rates and oxygen consumption.

If this depolarization mechanism proves to be true, it would have important clinical implications for the treatment of many disorders. Research on this topic, could help treating a wide array of illnesses ranging from stress and anxiety to respiratory, cardiac, sleep, and autonomic disorders. A common treatment for COPD is supplementation of oxygen which can lead to decreased respiratory drive 
in which the patient can enter respiratory failure, therefore further study of this possible mechanism could help to understand the underlying mechanisms of COPD [12]. The decrease in respiratory drive in COPD patients is no longer thought to be due to hypoxic drive but may be due to an increase in $\mathrm{CO}_{2}$ in the blood caused by the Haldane Effect and/or ventilation/perfusion imbalance [12], as previously discussed. However these mechanisms are still under debate [12] and levels of oxygen should not be ruled out as a factor involved in respiratory drive. We propose an additional respiratory drive mechanism in which widespread depolarization, due to low oxygen and increased sympathetic drive drives respiration. Further understanding of this possible mechanism could lead to the development of newer and more effective treatments for COPD and other disorders.

The proposed mechanism may also help to elucidate unexplained afflictions such as Sudden Infant Death Syndrome (SIDs). Abnormalities in the arcuate nucleus [89] and medulla oblongata [90] of the brainstem of SIDs victims suggests that they may have delayed development of cardiorespiratory control, arousal, and homeostatic responses. When breathing or other physiologic activity becomes compromised the infant does not arouse sufficiently to abate progression of such activity [91]. A recent study found that the infant brainstem is prone to the generation of hypoxic spreading depression. The study found a sudden depolarizing direct current of $-20 \mathrm{mV}$ spread from the spinal trigeminal nucleus to the hypoglossal nucleus, NTS, and the ventral respiratory group [92]. Research on subtle widespread depolarizations that may occur during decreased but not hypoxic levels of oxygen and increased sympathetic drive may give us insights into the causes of SIDs, as well as better means of prevention and preemptive treatments. This mechanism, if proven true, would have widespread clinical applications for many different disorders.

\section{Conclusion}

Up to this point, research has predominantly been focused on the influence of respiration on the brain, heart, ANS, or vasculature in isolated settings. The direct influence or correlation of respiration with brain activity, ANS function, heart activity, and BP levels demonstrate the notable control that respiration exerts in homeostatic physiology. We propose that cellular membrane potentials may also be influenced by respiration and that widespread partial hyperpolarization occurs during each inspiration and depolarization occurs during each expiration. We also propose that this widespread depolarization that occurs during expiration is a source of respiratory drive. The inclusion of membrane potential recordings in future research, as well as the direct investigation of respiration's role in membrane potential modification, may allow a better understanding of rhythmogenesis, the physiological impact of breathing techniques for normal health. Such knowledge could expand clinical options to supplement standard of care for many types of illness or disease, such as obstructive and non-obstructive breathing disorders, stress-related disorders, and abnormal sleep and further our understanding of the Hering-Breuer reflex and respiratory sinus arrhythmia.

\section{Conflict of interest}

There is no conflict of interest to report. No funding was received for the writing of this article.

\section{References}

[1] Pramanik T, Pudasaini B, Prajapati R. Immediate effect of a slow pace breathing exercise Bhramari pranayama on blood pressure and heart rate. Nepal Med Coll J 2010;12(3):154-7.
[2] Malpas SC. Neural influences on cardiovascular variability: possibilities and pitfalls. Am J Physiol Heart Circ Physiol 2002;282(1):H6-H20.

[3] Dergacheva O, Bateman R, Byrne P, Mendelowitz D. Orexinergic modulation of GABAergic neurotransmission to cardiac vagal neurons in the brain stem nucleus ambiguus changes during development. Neuroscience 2012;209:12-20.

[4] Que C-L, Kenyon CM, Olivenstein R, Macklem PT, Maksym GN. Homeokinesis and short-term variability of human airway caliber. J Appl Physiol 2001;91(3):1131-41.

[5] Binks AP, Bloch-Salisbury E, Banzett RB, Schwartzstein RM. Oscillation of the lung by chest-wall vibration. Respir Physiol 2001;126(3):245-9.

[6] Fiamma MN, Straus C, Thibault S, Wysocki M, Baconnier P, Similowski T. Effects of hypercapnia and hypocapnia on ventilatory variability and the chaotic dynamics of ventilatory flow in humans. Am J Physiol Regul Integr Comp Physiol 2007;292(5):R1985-93.

[7] Holt G, Johnson R, Davenport P. The transduction properties of intercostal muscle mechanoreceptors. BMC Physiol 2002;2(1):16.

[8] Hess A, Yu L, Klein I, et al. Neural mechanisms underlying breathing complexity. PLoS One 2013;8(10):e75740.

[9] Smith JC, Abdala AP, Rybak IA, Paton JF. Structural and functional architecture of respiratory networks in the mammalian brainstem. Philos Trans R Soc Lond B Biol Sci 2009;364(1529):2577-87.

[10] Spyer KM, Gourine AV. Chemosensory pathways in the brainstem controlling cardiorespiratory activity. Philos Trans $\mathrm{R}$ Soc Lond B Biol Sci 2009;364(1529):2603-10.

[11] Weil JV, Byrne-Quinn E, Sodal IE, et al. Hypoxic ventilatory drive in normal man. J Clin Invest 1970;49(6):1061-72.

[12] Kim V, Benditt JO, Wise RA, Sharafkhaneh A. Oxygen therapy in chronic obstructive pulmonary disease. Proc Am Thorac Soc 2008;5(4):513-8.

[13] Pattinson KT, Governo RJ, MacIntosh BJ, et al. Opioids depress cortical centers responsible for the volitional control of respiration. J Neurosci 2009;29(25):8177-86.

[14] Dempsey JA. New perspectives concerning feedback influences on cardiorespiratory control during rhythmic exercise and on exercise performance. J Physiol (Lond) 2012;590(Pt 17):4129-44.

[15] Prabhakar NR, Peng YJ. Peripheral chemoreceptors in health and disease. J Appl Physiol (1985) 2004;96(1):359-66.

[16] Talwar S, Agarwala S, Mittal CM, Choudhary SK, Airan B. Diaphragmatic palsy after cardiac surgical procedures in patients with congenital heart. Ann Pediatr Cardiol 2010:3(1):50-7.

[17] Jerath R, Harden K, Crawford M, Barnes VA, Jensen M. Role of cardiorespiratory synchronization and sleep physiology: effects on membrane potential in the restorative functions of sleep. Sleep Med 2014;15(3):279-88.

[18] Steriade M, Timofeev I. Neuronal plasticity in thalamocortical networks during sleep and waking oscillations. Neuron 2003;37(4):563-76.

[19] Lanfranchi PA, Fradette L, Gagnon JF, Colombo R, Montplaisir J. Cardiac autonomic regulation during sleep in idiopathic REM sleep behavior disorder. Sleep 2007;30(8):1019-25.

[20] Badra LJ, Cooke WH, Hoag JB, et al. Respiratory modulation of human autonomic rhythms. Am J Physiol Heart Circ Physiol 2001;280(6): H2674-88.

[21] Eckberg DL. The human respiratory gate. J Physiol 2003;548(Pt 2):339-52.

[22] Jerath R, Edry JW, Barnes VA, Jerath V. Physiology of long pranayamic breathing: neural respiratory elements may provide a mechanism that explains how slow deep breathing shifts the autonomic nervous system. Med Hypotheses 2006;67(3):566-71.

[23] Pal GK, Velkumary S, Madanmohan. Effect of short-term practice of breathing exercises on autonomic functions in normal human volunteers. Indian J Med Res 2004;120(2):115-21.

[24] Triposkiadis F, Karayannis G, Giamouzis G, Skoularigis J, Louridas G, Butler J The sympathetic nervous system in heart failure physiology, pathophysiology, and clinical implications. J Am Coll Cardiol 2009;54(19):1747-62.

[25] Goso Y, Asanoi H, Ishise H, et al. Respiratory modulation of muscle sympathetic nerve activity in patients with chronic heart failure. Circulation 2001:104(4):418-23.

[26] Seals DR, Suwarno NO, Dempsey JA. Influence of lung volume on sympathetic nerve discharge in normal humans. Circ Res 1990;67(1):130-41.

[27] Kubin L, Alheid GF, Zuperku EJ, McCrimmon DR. Central pathways of pulmonary and lower airway vagal afferents. J Appl Physiol 2006;101(2): 618-27.

[28] Moreira TS, Takakura AC, Colombari E, West GH, Guyenet PG. Inhibitory input from slowly adapting lung stretch receptors to retrotrapezoid nucleus chemoreceptors. J Physiol (Lond) 2007;580(1):285-300.

[29] Song HS, Lehrer PM. The effects of specific respiratory rates on heart rate and heart rate variability. Appl Psychophysiol Biofeedback 2003;28(1): 13-23.

[30] Sztajzel J. Heart rate variability: a noninvasive electrocardiographic method to measure the autonomic nervous system. Swiss Med Wkly 2004;134(3536):514-22.

[31] Yasuma F, Hayano J. Respiratory sinus arrhythmia: why does the heartbeat synchronize with respiratory rhythm? Chest 2004;125(2):683-90.

[32] Hanson B, Gill J, Western D, et al. Cyclical modulation of human ventricular repolarization by respiration. Front Physiol 2012;3:379.

[33] Bernardi L, Radaelli A, Solda PL, et al. Autonomic control of skin microvessels: assessment by power spectrum of photoplethysmographic waves. Clin Sci (Lond) 1996;90(5):345-55. 
[34] Eckberg DL. Point: counterpoint: respiratory sinus arrhythmia is due to a central mechanism vs. respiratory sinus arrhythmia is due to the baroreflex mechanism. J Appl Physiol 2009;106(5):1740-2 [discussion 1744].

[35] Zhang R, Zuckerman JH, Giller CA, Levine BD. Transfer function analysis of dynamic cerebral autoregulation in humans. Am J Physiol 1998;274(1 Pt 2):H233-41.

[36] Katura T, Tanaka N, Obata A, Sato H, Maki A. Quantitative evaluation of interrelations between spontaneous low-frequency oscillations in cerebral hemodynamics and systemic cardiovascular dynamics. Neuroimage 2006;31(4):1592-600.

[37] Pfurtscheller G, Daly I, Bauernfeind G, Muller-Putz GR. Coupling between intrinsic prefrontal $\mathrm{HbO} 2$ and central EEG beta power oscillations in the resting brain. PLoS One 2012;7(8):e43640.

[38] Strik C, Klose U, Kiefer C, Grodd W. Slow rhythmic oscillations in intracranial CSF and blood flow: registered by MRI. Acta Neurochir Suppl 2002;81: 139-42.

[39] Cang J, Isaacson JS. In vivo whole-cell recording of odor-evoked synaptic transmission in the rat olfactory bulb. J Neurosci 2003;23(10):4108-16.

[40] Briffaud V, Fourcaud-Trocme N, Messaoudi B, Buonviso N, Amat C. The relationship between respiration-related membrane potential slow oscillations and discharge patterns in mitral/tufted cells: what are the rules? PLoS One 2012;7(8):e43964.

[41] Phillips ME, Sachdev RN, Willhite DC, Shepherd GM. Respiration drives network activity and modulates synaptic and circuit processing of lateral inhibition in the olfactory bulb. J Neurosci 2012;32(1):85-98.

[42] Guyenet PG, Stornetta RL, Abbott SB, Depuy SD, Kanbar R. The retrotrapezoid nucleus and breathing. Adv Exp Med Biol 2012;758:115-22.

[43] Nattie EE. The retrotrapezoid nucleus and the 'drive' to breathe. J Physiol (Lond) 2006;572(2) [311-311].

[44] Takakura ACT, Moreira TS, Colombari E, West GH, Stornetta RL, Guyenet PG Peripheral chemoreceptor inputs to retrotrapezoid nucleus (RTN) CO2sensitive neurons in rats. J Physiol (Lond) 2006;572(2):503-23.

[45] Stornetta RL, Moreira TS, Takakura AC, et al. Expression of Phox $2 b$ by brainstem neurons involved in chemosensory integration in the adult rat. Neurosci 2006;26(40):10305-14.

[46] Onimaru H, Homma I. Point:counterpoint: the parafacial respiratory group (pFRG)/pre-Bötzinger complex (preBötC) is the primary site of respiratory rhythm generation in the mammal. J Appl Physiol 2006;100(6):2094-8.

[47] Matsumoto S, Ikeda M, Nishikawa T, Tanimoto T, Yoshida S, Saiki C. Inhibitory mechanism of slowly adapting pulmonary stretch receptors after release from hyperinflation in anesthetized rabbits. Life Sci 2000;67(12):1423-33.

[48] Schelegle ES. Functional morphology and physiology of slowly adapting pulmonary stretch receptors. Anat Rec A Discov Mol Cell Evol Biol 2003;270(1):11-6.

[49] Ezure K, Tanaka I, Saito Y, Otake K. Axonal projections of pulmonary slowly adapting receptor relay neurons in the rat. J Comp Neurol 2002;446(1):81-94.

[50] Otake K, Nakamura Y, Tanaka I, Ezure K. Morphology of pulmonary rapidly adapting receptor relay neurons in the rat. J Comp Neurol 2001;430(4):458-70.

[51] Berger AJ. Dorsal respiratory group neurons in the medulla of cat: spinal projections, responses to lung inflation and superior laryngeal nerve stimulation. Brain Res 1977;135(2):231-54.

[52] Kohl J, Koller EA. Heterogeneous activity of pulmonary vagal receptors during high-frequency oscillation ventilation. Lung 1995;173(5):281-90.

[53] Wozniak JA, Davenport PW, Kosch PC. Responses of pulmonary vagal mechanoreceptors to high-frequency oscillatory ventilation. J Appl Physiol 1988;65(2):633-9.

[54] Chouhan AK, Ivannikov MV, Lu Z, Sugimori M, Llinas RR, Macleod GT. Cytosolic calcium coordinates mitochondrial energy metabolism with presynaptic activity. J Neurosci 2012;32(4):1233-43.

[55] Gutterman DD, Cowley Jr AW. Relating cardiac performance with oxygen consumption: historical observations continue to spawn scientific discovery Am J Physiol Heart Circ Physiol 2006;291(6):H2555-6.

[56] Rolfe DF, Brown GC. Cellular energy utilization and molecular origin of standard metabolic rate in mammals. Physiol Rev 1997:77(3):731-58.

[57] Burton DA, Stokes K, Hall GM. Physiological effects of exercise. Contin Educ Anaesth Crit Care \& Pain 2004;4(6):185-8.

[58] Boutilier RG. Mechanisms of cell survival in hypoxia and hypothermia. J Exp Biol 2001;204(Pt 18):3171-81.

[59] Alberts B, Johnson A, Lewis J, Raff M, Roberts K, Walter P. Electron-transport chains and their proton pumps. In: Molecular biology of the cell. New York: Garland Science; 2002.

[60] Alberts B, Johnson A, Lewis J, Raff M, Roberts K, Walter P. The mitochondrion. In: Molecular biology of the cell. New York: Garland Science; 2002.

[61] Perry SW, Norman JP, Barbieri J, Brown EB, Gelbard HA. Mitochondria membrane potential probes and the proton gradient: a practical usage guide. Biotechniques 2011;50(2):98-115.

[62] Brunori M, Antonini G, Malatesta F, Sarti P, Wilson MT. Cytochrome-c oxidase. Eur J Biochem 1987;169(1):1-8.
[63] Buckman JF, Reynolds IJ. Spontaneous changes in mitochondrial membrane potential in cultured neurons. J Neurosci 2001;21(14):5054-65.

[64] Ichas F, Jouaville LS, Mazat JP. Mitochondria are excitable organelles capable of generating and conveying electrical and calcium signals. Cell 1997:89(7):1145-53.

[65] Huser J, Blatter LA. Fluctuations in mitochondrial membrane potential caused by repetitive gating of the permeability transition pore. Biochem J 1999;343(Pt 2):311-7.

[66] Duchen MR, Leyssens A, Crompton M. Transient mitochondrial depolarizations reflect focal sarcoplasmic reticular calcium release in single rat cardiomyocytes. J Cell Biol 1998;142(4):975-88.

[67] Krippeit-Drews P, Dufer M, Drews G. Parallel oscillations of intracellular calcium activity and mitochondrial membrane potential in mouse pancreatic B-cells. Biochem Biophys Res Commun 2000;267(1):179-83.

[68] Bonnet S, Archer SL, Allalunis-Turner J, et al. A mitochondria-K+ channel axis is suppressed in cancer and its normalization promotes apoptosis and inhibits cancer growth. Cancer Cell 2007;11(1):37-51.

[69] Chandel NS, Budinger GRS, Choe SH, Schumacker PT. Cellular respiration during hypoxia: role of cytochrome oxidase as the oxygen sensor in hepatocytes. J Biol Chem 1997;272(30):18808-16.

[70] Di Lisa F, Menabo R, Canton M, Petronilli V. The role of mitochondria in the salvage and the injury of the ischemic myocardium. Biochim Biophys Acta 1998;1366(1-2):69-78.

[71] Kaim G, Dimroth P. Voltage-generated torque drives the motor of the ATP synthase. EMBO J 1998;17(20):5887-95.

[72] Colleoni F, Padmanabhan N, Yung HW, et al. Suppression of mitochondrial electron transport chain function in the hypoxic human placenta: a role for miRNA-210 and protein synthesis inhibition. PLoS One 2013;8(1):e55194.

[73] Hyllienmark L, Brismar T. Effect of hypoxia on membrane potential and resting conductance in rat hippocampal neurons. Neuroscience 1999;91(2):511-7.

[74] Plant LD, Kemp PJ, Peers C, Henderson Z, Pearson HA. Hypoxic depolarization of cerebellar granule neurons by specific inhibition of TASK-1. Stroke 2002;33(9):2324-8.

[75] Bahar S, Fayuk D, Somjen GG, Aitken PG, Turner DA. Mitochondrial and intrinsic optical signals imaged during hypoxia and spreading depression in rat hippocampal slices. J Neurophysiol 2000;84(1):311-24.

[76] Bahar S, Fayuk D, Somjen GG, Aitken PG, Turner DA. Mitochondrial and Intrinsic Optical Signals Imaged During Hypoxia and Spreading Depression in Rat Hippocampal Slices. J Neurophysiol 2000;84(1):311-24.

[77] Parkes MJ. Breath-holding and its breakpoint. Exp Physiol 2006:91(1):1-15.

[78] Dujic Z, Uglesic L, Breskovic T, et al. Involuntary breathing movements improve cerebral oxygenation during apnea struggle phase in elite divers. J Appl Physiol 2009;107(6):1840-6.

[79] Dempsey JA, Veasey SC, Morgan BJ, O’Donnell CP. Pathophysiology of sleep apnea. Physiol Rev 2010;90(1):47-112.

[80] Younes M. Role of respiratory control mechanisms in the pathogenesis of obstructive sleep disorders. J Appl Physiol 2008;105(5):1389-405.

[81] Cutler MJ, Swift NM, Keller DM, Wasmund WL, Smith ML. Hypoxia-mediated prolonged elevation of sympathetic nerve activity after periods of intermittent hypoxic apnea. J Appl Physiol (1985) 2004;96(2):754-61.

[82] Leuenberger UA, Brubaker D, Quraishi S, Hogeman CS, Imadojemu VA, Gray KS. Effects of intermittent hypoxia on sympathetic activity and blood pressure in humans. Auton Neurosci 2005;121(1-2):87-93.

[83] Aitken PG, Jing J, Young J, Somjen GG. Ion channel involvement in hypoxiainduced spreading depression in hippocampal slices. Brain Res 1991;541(1): $7-11$.

[84] Takano T, Tian GF, Peng W, et al. Cortical spreading depression causes and coincides with tissue hypoxia. Nat Neurosci 2007;10(6):754-62.

[85] Ito N, Fukumoto S. Symptoms and management of tetany. Clin Calcium 2007;17(8):1234-9.

[86] Axelrod DR. Organic acids and calcium in hyperventilation. J Appl Physiol 1961;16(4):709-12.

[87] Chin K, Ohi M, Kuno K. Hyperventilation syndrome. Nihon Kyobu Shikkan Gakkai Zasshi 1992;30(Suppl.):147-53.

[88] Ames F. The hyperventilation syndrome. Br J Psychiatry 1955;101(424): 466-525.

[89] Panigrahy A, Filiano JJ, Sleeper LA, et al. Decreased kainate receptor binding in the arcuate nucleus of the sudden infant death syndrome. J Neuropathol Exp Neurol 1997;56(11):1253-61.

[90] Panigrahy A, Filiano J, Sleeper LA, et al. Decreased serotonergic receptor binding in rhombic lip-derived regions of the medulla oblongata in the sudden infant death syndrome. J Neuropathol Exp Neurol 2000;59(5):377-84.

[91] Changing concepts of sudden infant death syndrome: implications for infant sleeping environment and sleep position. American Academy of Pediatrics. Task Force on Infant Sleep Position and Sudden Infant Death Syndrome. Pediatrics, 2000;105(3 Pt 1):650-656.

[92] Funke F, Kron M, Dutschmann M, Muller M. Infant brain stem is prone to the generation of spreading depression during severe hypoxia. J Neurophysiol 2009;101(5):2395-410. 